\title{
Converging to and escaping from the global equilibrium: Isostables and optimal control
}

\author{
Alexandre Mauroy
}

\begin{abstract}
This paper studies the optimal control of trajectories converging to or escaping from a stable equilibrium. The control duration is assumed to be short. When the control is turned off, the trajectories have not reached the target and they subsequently evolve according to the free motion dynamics. In this context, we show that the problem can be formulated as a finite-horizon optimal control problem which relies on the notion of isostables. For linear and nonlinear systems, we solve this problem using Pontryagin's maximum principle and we study the relationship between the optimal solutions and the geometry of the isostables. Finally, optimal strategies for choosing the magnitude and duration of the control are considered.
\end{abstract}

\section{INTRODUCTION}

Optimal control techniques have been widely used for time-optimal steering of systems toward the equilibrium (see e.g. [1], [6], [11]). In this context, it is usually assumed that the control effectively drives the trajectory toward the desired target, however long it takes. In some situations, however, the control duration is limited (e.g. limited amount of available energy, etc.) and the controlled trajectory may not have reached the equilibrium when the control is turned off. This is even so when the initial condition is far from the target or when the control is weak. In this case, the time-optimal control problem is irrelevant. More importantly, it is not straightforward to formulate an optimal control problem which optimizes the (infinite-time) convergence of a trajectory whose asymptotic behavior is governed by the free motion dynamics. For instance, choosing a neighborhood of the equilibrium as alternative target set is not convenient, since the obtained results would depend on the size of the neighborhood. A similar issue arises when considering the optimal (finite-duration) escape from a stable equilibrium with a large basin of attraction.

In this paper, we propose a relevant formulation for the above problem (i.e. convergence to/escape from the equilibrium) which is adapted to the short duration of the control. In particular, we show that the relevant end cost function for the problem- to be maximized when the control is switched off-is based on the notion of isostables. Introduced in our recent work [5] (see also [10] for slow-fast systems), the isostables are sets of the state space that capture the asymptotic behavior of the uncontrolled system. They provide a unique and rigorous measure of how far-with respect to time-the trajectory is from the equilibrium. Note that the mere distance to the equilibrium is not a good measure since

A. Mauroy is with the Department of Electrical Engineering and Computer Science, University of Liège, 4000 Liège, Belgium a.mauroy@ulg.ac.be the trajectory evolves according to a (possibly nonlinear) system. The isostables are also related to the global stability properties of the equilibrium [4], and it is therefore natural to use them for the optimal control problem.

We assume that the control is a vector of constant magnitude, thereby considering a steering control problem (e.g. vehicles moving in a velocity field [12]). Although the optimal control problem cannot be solved analytically, the optimal control direction is directly related to the geometry of the isostables in some particular cases. In addition, we show that the best results are obtained with a weak and long control, in which case the optimal control is perpendicular to the isostables (local control).

The paper is organized as follows. The concept of isostables and its related Koopman operator framework are introduced in Section II. In Section III, we present the optimal control problem that we solve for both linear and nonlinear systems, using Pontryagin's maximum principle. Section IV discusses the optimal strategies when the duration and magnitude of the control can be chosen. Finally, we give some concluding remarks in Section V.

\section{PRELIMINARIES: ISOSTABLES AND KOOPMAN OPERATOR}

The isostables (of a stable equilibrium) are defined in our previous work [5] as particular sets related to the asymptotic behavior of the trajectories. (Note that a similar notion was introduced in [10] in the particular case of slow-fast systems.) In this section, we review the concept of isostables for both linear and nonlinear systems and we highlight their relationship with the so-called Koopman operator. The interested reader may refer to [5] for more details on the isostables and their properties.

\section{A. Linear systems}

Consider the stable linear system

$$
\dot{x}=A x, \quad x \in \mathbb{R}^{n}
$$

where the real matrix $A$ has $n$ distinct eigenvalues $\lambda_{j}$ with $\Re\left\{\lambda_{j}\right\} \leq \Re\left\{\lambda_{1}\right\}<0$ for all $j$, associated with the right and left eigenvectors $v_{i}$ and $\tilde{v}_{j}$ respectively. We assume that the eigenvectors are normalized so that $\left(v_{j} \cdot \tilde{v}_{j}\right)=1$, where denotes the inner product in $\mathbb{C}^{n}$. The solution $\varphi^{t}(x)$ of (1) for the initial condition $x$-i.e., the flow map-is given by

$$
\varphi^{t}(x)=\sum_{j=1}^{n}\left(x \cdot \tilde{v}_{j}\right) v_{j} e^{\lambda_{j} t} .
$$


Since the first term is dominant over the other terms as $t \rightarrow$ $\infty$, the function $\phi(x) \triangleq x \cdot \tilde{v}_{1}$ is sufficient to characterize the asymptotic behavior of the trajectory starting at $x$. In [5], the isostables of a linear system are defined as the level sets of $|\phi(x)|=\left|x \cdot \tilde{v}_{1}\right|$. They provide a measure of "how long" the trajectory will take to converge toward the origin (see the properties below). For two-dimensional linear systems, each isostable corresponds to a pair of straight lines parallel to $v_{2}$ (when $\lambda_{1}$ is real) or to an ellipse centered at the origin (when $\lambda_{1}$ is complex).

\section{B. Nonlinear systems and Koopman operator}

We will now define the notion of isostables for the nonlinear system

$$
\dot{x}=F(x), \quad x \in \mathbb{R}^{n}
$$

which admits a stable equilibrium $\hat{x}$-i.e., $F(\hat{x})=0$-with a basin of attraction $\mathcal{B}(\hat{x}) \subseteq \mathbb{R}^{n}$. We assume that the vector field $F$ is continuously differentiable and that the Jacobian matrix $J(\hat{x})$ at the equilibrium satisfies the same properties as the matrix $A$ in (1).

As a preliminary to a general definition of the isostables, we introduce the so-called Koopman operator.

Definition 1 (Koopman operator): For a given functional space $\mathcal{F}$, the (semi-)group of Koopman operators $U^{t}: \mathcal{F} \rightarrow$ $\mathcal{F}$ associated with a flow map $\varphi^{t}$ is given by

$$
U^{t} f=f \circ \varphi^{t}, \quad f \in \mathcal{F}, t \in \mathbb{R} .
$$

Through the Koopman operator framework, the notion of isostables can be extended to nonlinear systems. We have the following general definition [5].

Definition 2 (Isostables): For the system (2), the isostables are the level sets of $|\phi(x)|$, where the function $\phi(x)$ satisfies:

1. $\phi$ is $C^{1}$ in the neighborhood of the equilibrium $\hat{x}$;

2. $\phi$ is an eigenfunction of the Koopman operator, associated with the eigenvalue $\lambda_{1}$, i.e.

$$
U^{t} \phi=\phi \circ \varphi^{t}(x)=e^{\lambda_{1} t} \phi(x) .
$$

Through Definition 2, it can be shown that the isostables are unique. This definition is also in agreement with the case of linear systems: it is easy to verify that the function $\phi(x)=$ $x \cdot \tilde{v}_{1}$ is a $C^{1}$ function that satisfies (3).

The isostables capture the asymptotic behavior of the trajectories (of the unforced system) and provide a rigorous and unique measure of the time required by the trajectories to reach a close neighborhood of the equilibrium. In particular, (3) implies the following properties.

Property 1: Two trajectories (of the unforced system) with initial conditions on the same isostable simultaneously intersect the successive isostables of decreasing value and approach the equilibrium $\hat{x}$ synchronously. Note that $\phi(\hat{x})=$ 0 .

Property 2: If two initial conditions $x_{1}$ and $x_{2}$ belong to different isostables, e.g. $\left|\phi\left(x_{1}\right)\right|<\left|\phi\left(x_{2}\right)\right|$, then the corresponding trajectories (of the unforced system) intersect the same isostables with a constant time delay

$$
\Delta t=\frac{\ln \left|\phi\left(x_{1}\right)\right|-\ln \left|\phi\left(x_{2}\right)\right|}{\Re\left\{\lambda_{1}\right\}} .
$$

In particular, they approach the neighborhood of the equilibrium (i.e. a level set $|\phi(x)|=C \ll 1$ ) with the time delay $\Delta t$.

Remark 1: If the flow $\varphi^{t}$ is induced by (2), the function $f(t, x)=U^{t} f_{0}(x), f_{0} \in C^{1}\left(\mathbb{R}^{n}\right)$, satisfies (see e.g. [3])

$$
\frac{\partial f}{\partial t}=F \cdot \nabla_{x} f
$$

with the initial condition $f(0, x)=f_{0}(x)$ and with the gradient $\nabla_{x} f=\left(\partial f / \partial x_{1}, \ldots, \partial f / \partial x_{n}\right)$. It follows that the $\phi$ is solution of the partial differential equation

$$
\lambda_{1} \phi=F \cdot \nabla_{x} \phi .
$$

Numerical computation: The isostables can be computed by using Laplace averages [7] evaluated along the trajectories. More precisely, the eigenfunction $\phi(x)$ is given by

$$
\phi(x)=\lim _{T \rightarrow \infty} \frac{1}{T} \int_{0}^{T} f \circ \varphi^{t}(x) e^{-\lambda_{1} t} d t
$$

for any function $f \in C^{1}(\hat{x})$ which satisfies $f(\hat{x})=0$ and $\left|\nabla_{x} f(\hat{x}) \cdot v_{1}\right|=1$. This method yields numerical schemes that are efficient to compute the isostables, even in highdimensional spaces (see [5] for more details).

\section{OPTIMAL CONTROL PROBLEM}

With the concept of isostables, we are now in position to write the statement of the optimal control problem. Our goal is to design a control input which is applied to the system (2) during a short time interval $[0, T]$. (We assume that the control input cannot start at some time $t>0$.) The optimal control, related to the optimal trajectory $x^{*}(t), t \in$ $[0, T]$, should minimize (optimal convergence problem) or maximize (optimal escape problem) the time required by the subsequent free motion $\varphi^{t-T}\left(x^{*}(T)\right), t \in[T, \infty)$, to reach a close neighborhood of the equilibrium. In other words, the optimal trajectory $x^{*}(t)$ is the admissible trajectory which is characterized by the maximum time delay (4) with respect to a (virtual) trajectory $\varphi^{t}\left(x_{0}\right)$ of the uncontrolled system, starting at the same initial condition $x_{0}$. Property 2 with the initial conditions $x_{1}=x^{*}(T)$ and $x_{2}=\varphi^{T}\left(x_{0}\right)$ implies that this optimal time delay is given by

$$
\begin{aligned}
\Delta t^{*} & =\left|\frac{\ln \left|\phi\left(x^{*}(T)\right)\right|-\ln \left|\phi\left(\varphi^{T}\left(x_{0}\right)\right)\right|}{\Re\left\{\lambda_{1}\right\}}\right| \\
& =\left|\frac{\ln \left|\phi\left(x^{*}(T)\right)\right|-\ln \left|\phi\left(x_{0}\right)\right|}{\Re\left\{\lambda_{1}\right\}}-T\right|
\end{aligned}
$$

where we used (3). It is clear that the time delay (8) is maximum provided that the optimal trajectory is the admissible trajectory that reaches at time $T$ the isostable with the lowest value $|\phi|$ (optimal convergence problem) or the highest value $|\phi|$ (optimal escape problem).

Since we assume that the input $u(t)$ has constant magnitude $\|u(t)\|=\bar{u}$, for all $t \in[0, T]$, we only have to compute 
its orientation $e(t)$, with $\|e(t)\|=1$ (steering problem). Finally, we can formulate the optimal control problem as follows.

The optimal control problem: For $x_{0} \in \mathcal{B}(\hat{x}), \bar{u} \in \mathbb{R}^{+}$, and $T \in \mathbb{R}^{+}$, find the time-dependent vector $e^{*}(t)=$ $[0, T] \rightarrow \mathbb{S}^{n}$ such that

$$
e^{*}(t)= \begin{cases}\operatorname{argmin}_{\|e(t)\|=1}\left|\phi\left(\varphi_{\bar{u}}^{T}\left(x_{0}, e(t)\right)\right)\right| & \text { convergence } \\ \operatorname{argmax}_{\|e(t)\|=1}\left|\phi\left(\varphi_{\bar{u}}^{T}\left(x_{0}, e(t)\right)\right)\right| & \text { escape }\end{cases}
$$

where $\varphi_{\bar{u}}^{t}\left(x_{0}, e(t)\right)$ is the solution of

$$
\dot{x}=F(x)+\bar{u} e(t)
$$

with the initial condition $\varphi_{\bar{u}}^{0}\left(x_{0}, e(t)\right)=x_{0}$.

This problem corresponds to a finite-horizon optimal control problem with no cost-to-go function and with the end cost function $g(x)= \pm|\phi(x)|$ (to be maximized). From this point on, we consider that the symbol \pm is a positive sign for the optimal escape problem and a negative sign for the optimal convergence problem. The end cost function is directly related to the isostables and can be computed through the Laplace average method (7).

Remark 2: For the optimal convergence problem, the initial condition $x_{0}$ is assumed to be far from the equilibrium $\hat{x}$ (or equivalently $\bar{u}$ and $T$ are assumed to be sufficiently small) so that the control cannot drive the trajectory to the equilibrium within the time interval $[0, T]$. Otherwise, the proposed optimal control problem based on the notion of isostables is irrelevant and should be replaced by a classic time-optimal control problem [6]. Similarly, for the optimal escape problem, we assume that the control cannot drive the trajectory out of the basin of attraction $\mathcal{B}(\hat{x})$.

\section{A. Local (suboptimal) control}

A naive solution $e^{(*)}(t)$ to the optimal control problem can be obtained directly from the mere knowledge of the isostables. At each time $t \in[0, T]$, a local approximation of $\phi$ is computed through the Laplace average (7) and the control follows the steepest descent/ascent policy

$$
e^{(*)}(t)= \pm \frac{\nabla_{x}\left|\phi\left(x^{(*)}(t)\right)\right|}{\left\|\nabla_{x}\left|\phi\left(x^{(*)}(t)\right)\right|\right\|}
$$

with $x^{(*)}(t)=\varphi_{\bar{u}}^{t}\left(x_{0}, e^{(*)}(t)\right)$. In this case, $e^{(*)}(t)$ is always perpendicular to the isostables along the trajectory.

Except in particular situations (see below), this "local" control is suboptimal. However, it is the most optimal control that can be found if only a local approximation of the isostables is known or if the vector field $F$ is unknown. (Note that the computation of the Laplace averages (7) can be performed even if the vector field is unknown.) The interest of this local control is also demonstrated in Section IV.

\section{B. Solving the optimal control problem}

If the geometry of the isostables is known in the whole basin of attraction, we can apply usual optimal control methods to solve our optimal control problem (see e.g. the monograph [2] for a review of the optimal control methods).
1) Pontryagin's maximum principle: For the system (9), the Hamiltonian is given by

$$
H(x, p, e)=p \cdot(F(x)+\bar{u} e)
$$

where $p \in \mathbb{R}^{n}$ is the costate. The optimal trajectory $x^{*}(t)=$ $\varphi_{\bar{u}}^{t}\left(x_{0}, e^{*}(t)\right)$ and the optimal control $e^{*}(t)$ satisfy, for all $t \in[0, T]$,

$\dot{x}^{*}(t)=\nabla_{p} H\left(x^{*}(t), p^{*}(t), e^{*}(t)\right)=F\left(x^{*}(t)\right)+\bar{u} e^{*}(t)$

$\dot{p}^{*}(t)=-\nabla_{x} H\left(x^{*}(t), p^{*}(t), e^{*}(t)\right)=-J^{T}\left(x^{*}(t)\right) p^{*}(t)$

for some costate function $p^{*}(t):[0, T] \rightarrow \mathbb{R}^{n}$ and where $J^{T}(x)$ is the transposed Jacobian matrix of $F$ at $x$. In addition, we have the terminal condition

$$
p^{*}(T)=\nabla_{x} g\left(x^{*}(T)\right)= \pm \nabla_{x}\left|\phi\left(x^{*}(T)\right)\right| .
$$

From Pontryagin's maximum principle [9], it follows that

$$
e^{*}(t)=\operatorname{argmax}_{\|e(t)\|=1} H\left(x^{*}(t), p^{*}(t), e(t)\right)=\frac{p^{*}(t)}{\left\|p^{*}(t)\right\|},
$$

so that the optimal control is parallel to the costate.

The optimal control problem is solved as follows: (i) compute the function $\phi$ on $\mathcal{B}(\hat{x})$ using the Laplace averages (7) and (ii) solve

$$
\begin{aligned}
\dot{x}^{*} & =F\left(x^{*}\right)+\bar{u} p^{*} /\left\|p^{*}\right\| \\
\dot{p}^{*} & =-J^{T}\left(x^{*}\right) p^{*}
\end{aligned}
$$

with the final costate (11) and the initial state $x^{*}(0)=x_{0}$. Note that (11) and (12) imply that the final control value $e^{*}(T)$ is perpendicular to the isostable at $x^{*}(T)$.

Remark 3: Taking the gradient of (6), we have

$$
F \cdot \nabla_{x} \phi=-J^{T} \nabla_{x} \phi+\lambda_{1} \nabla_{x} \phi
$$

where the left hand side corresponds to the time derivative of $\nabla_{x} \phi$ along the trajectory $\varphi^{t}(x)$ of the unforced system. This implies that $\nabla_{x} \phi\left(\varphi^{t}(x)\right)$ is parallel to the vector $\tilde{p}$ which is solution of

$$
\dot{\tilde{p}}=-J^{T}\left(\varphi^{t}(x)\right) \tilde{p}
$$

with $\tilde{p}(0)=\nabla_{x} \phi(x)$. It is remarkable that the dynamics (14) (for the costate $p^{*}$ ) and (15) (for the gradient $\nabla_{x} \phi$ ) are identical, except that they hold on two different trajectories (resp. $\varphi^{t}(x)$ and $x^{*}(t)$ ). This explains why the optimal control (12) is usually not the local control (10) perpendicular to the isostables (see Section III-A).

2) Hamilton-Jacobi-Bellman equation: Although we solve the optimal control problem using the Pontryagin's maximum principle as described above, we briefly introduce the Hamilton-Jacobi-Bellman (HJB) equation. The (continuously differentiable) value function $v:[0, T] \times \mathcal{B}(\hat{x}) \rightarrow \mathbb{R}$ is defined by

$$
\begin{aligned}
v(t, x) & =\sup _{\|e(t)\|=1} g\left(\varphi_{\bar{u}}^{T-t}(x, e(t))\right) \\
& =\sup _{\|e(t)\|=1} \pm\left|\phi\left(\varphi_{\bar{u}}^{T-t}(x, e(t))\right)\right| .
\end{aligned}
$$


The value function is solution of the HJB equation

$$
\begin{aligned}
\frac{\partial v}{\partial t} & =-\max _{\|e\|=1}\left\{(F+\bar{u} e) \cdot \nabla_{x} v\right\} \\
& =-F \cdot \nabla_{x} v-\bar{u}\left\|\nabla_{x} v\right\|
\end{aligned}
$$

with the final condition $v(T, x)= \pm|\phi(x)|$. When (16) is solved, the optimal trajectory $x^{*}(t)$ can be obtained with the dynamic programming method . Note that $\nabla_{x} v\left(t, x^{*}(t)\right)=$ $p^{*}(t)$ and $e^{*}(t)=\nabla_{x} v\left(t, x^{*}(t)\right) /\left\|\nabla_{x} v\left(t, x^{*}(t)\right)\right\|$.

Remark 4 (HJB equation and Koopman operator): It is noticeable that the HJB equation (16) is very similar to the Koopman operator equation (5). Through the change of variable $t^{\prime}=T-t$, (16) becomes

$$
\frac{\partial v}{\partial t^{\prime}}=F \cdot \nabla_{x} v+\bar{u}\left\|\nabla_{x} v\right\|,
$$

with the initial condition $v\left(t^{\prime}=0, x\right)= \pm|\phi(x)|$. It corresponds to (5) with the additional nonlinear term $\bar{u}\left\|\nabla_{x} v\right\|$.

\section{Weak and strong control}

Even for simple dynamics, it is generally impossible to find an (analytic) expression of the optimal control $e^{*}(t)$ that is directly derived from the function $\phi$ (i.e. from the isostables). However, in case of weak or strong inputs, the optimal control can be easily obtained from the mere knowledge of the isostables.

1) Weak control: When the control magnitude is very small with respect to the vector field, i.e. $\bar{u} \ll\|F\|$, we can neglect the nonlinear term in (17), so that the (backward) HJB equation is equivalent to the action of the Koopman operator $U^{t}$ (see Remark 4). It follows that

$$
v\left(t^{\prime}, x\right) \approx U^{t^{\prime}} v\left(t^{\prime}=0, x\right)= \pm U^{t^{\prime}}|\phi(x)|= \pm\left|e^{\lambda_{1} t^{\prime}}\right||\phi(x)|
$$

with $t^{\prime}=T-t$ and where we used (3). This implies that the optimal control is given by

$$
e^{*}(t)=\frac{\nabla_{x} v\left(t, x^{*}(t)\right)}{\left\|\nabla_{x} v\left(t, x^{*}(t)\right)\right\|} \approx \pm \frac{\nabla_{x}\left|\phi\left(x^{*}(t)\right)\right|}{\left\|\nabla_{x}\left|\phi\left(x^{*}(t)\right)\right|\right\|}
$$

and corresponds to the local control (10) described in Section III-A. In this case, $e^{*}(t)$ is perpendicular to the isostables not only at time $T$ as imposed by the endpoint cost (11), but also at any time $t<T$. This result can also be obtained through the discussion in Remark 3 and by observing that $\varphi^{t}(x) \approx x^{*}(t)$.

2) Strong control: When the control magnitude is very large with respect to the vector field, i.e. $\bar{u} \gg\|F\|$, (9) becomes

$$
\dot{x} \approx \bar{u} e(t)
$$

and it is well-known that the optimal control is a constant vector $e^{*}(t)=e^{*}$ with

$$
e^{*}=\operatorname{argmax}_{\|e\|=1} \pm\left|\phi\left(x_{0}+\bar{u} T e\right)\right| .
$$

In this case, the optimal direction $e^{*}$ can be determined directly from the global geometry of the isostables.

\section{Examples: linear and nonlinear systems}

1) Linear system with stable sink: Consider the linear system (1) where $\lambda_{1}$ is real. We have $\phi(x)=x \cdot \tilde{v}_{1}$ and $\nabla_{x}|\phi|=\operatorname{sign}\left(x \cdot \tilde{v}_{1}\right) \tilde{v}_{1}$. The costate dynamics (14) is $\dot{p}^{*}=-A^{T} p^{*}$ and (11) yields $p^{*}(T)= \pm \operatorname{sign}\left(x^{*}(T) \cdot \tilde{v}_{1}\right) \tilde{v}_{1}$. It follows that

$$
p^{*}(t)= \pm e^{\lambda_{1}(T-t)} \operatorname{sign}\left(x^{*}(T) \cdot \tilde{v}_{1}\right) \tilde{v}_{1}
$$

and (12) implies that the optimal control is

$$
e^{*}(t)= \pm \operatorname{sign}\left(x_{0} \cdot \tilde{v}_{1}\right) \frac{\tilde{v}_{1}}{\left\|\tilde{v}_{1}\right\|},
$$

where we used $\operatorname{sign}\left(x^{*}(T) \cdot \tilde{v}_{1}\right)=\operatorname{sign}\left(x_{0} \cdot \tilde{v}_{1}\right)$ since we assume that the optimal trajectory does not reach the isostable $x \cdot \tilde{v}_{1}=0$. The optimal control $e^{*}(t)$ is constant and perpendicular to the isostables. (The isostables are parallel lines.) It corresponds to the local control (10) described in Section III-A.

The optimal delay (8) can be computed analytically. We have

$$
\begin{aligned}
\frac{d \phi}{d t}\left(x^{*}(t)\right) & =\nabla_{x} \phi\left(x^{*}(t)\right) \cdot\left(F\left(x^{*}(t)\right)+\bar{u} e^{*}(t)\right) \\
& =\lambda_{1} \phi\left(x^{*}(t)\right) \pm \bar{u} \operatorname{sign}\left(x_{0} \cdot \tilde{v}_{1}\right)
\end{aligned}
$$

where we used (6), (19), and $\nabla_{x} \phi=\tilde{v}_{1}$. This implies that

$$
\phi\left(x^{*}(T)\right)=e^{\lambda_{1} T}\left(\phi\left(x_{0}\right) \pm \frac{\bar{u} \operatorname{sign} \phi\left(x_{0}\right)}{\lambda_{1}}\right) \mp \frac{\bar{u} \operatorname{sign} \phi\left(x_{0}\right)}{\lambda_{1}}
$$

with $\phi\left(x_{0}\right)=x_{0} \cdot \tilde{v}_{1}$ and the optimal delay $\Delta t^{*}$ is given by (8).

2) Linear system with stable focus: In the case of a linear system (1) with $\lambda_{1}$ complex, the optimal control problem is more complicated. It follows from (13)-(14) that

$$
\dot{x}^{*}=A x^{*}+\bar{u} \frac{e^{-A^{T} t} p^{*}(0)}{\left\|e^{-A^{T} t} p^{*}(0)\right\|}
$$

for some $p^{*}(0)$ so that we have

$$
x^{*}(T)=e^{A T}\left(x_{0}+\bar{u} I\left(p^{*}(0)\right)\right)
$$

with the vector-valued function

$$
I(p)=\int_{0}^{T} \frac{e^{-A \tau} e^{-A^{T} \tau} p}{\left\|e^{-A^{T} t} p\right\|} d \tau .
$$

Since $\phi\left(x^{*}(T)\right)=x^{*}(T) \cdot \tilde{v}_{1}$, it follows that

$$
p^{*}(0)=\operatorname{argmax}_{\|p\|=1} \pm\left|e^{A T}\left(x_{0}+\bar{u} I(p)\right) \cdot \tilde{v}_{1}\right|
$$

and the optimal control is given by (12) with $p^{*}(t)=$ $\exp \left(-A^{T} t\right) p^{*}(0)$. Optimal trajectories are illustrated in Fig. 1.

In contrast to the linear case with $\lambda_{1}$ real, the optimal control is not perpendicular to the isostables (see e.g. the red optimal trajectory in Fig. 1). This is explained by the fact that the gradient $\nabla_{x}|\phi(x)|=\left|x \cdot \tilde{v}_{1}\right|$ is not constant since $\tilde{v}_{1}$ is a complex-valued vector. The function $\phi$ has a nonzero curvature (nonzero Hessian) and the isostables-i.e., ellipses centered at the origin — are characterized by regions 


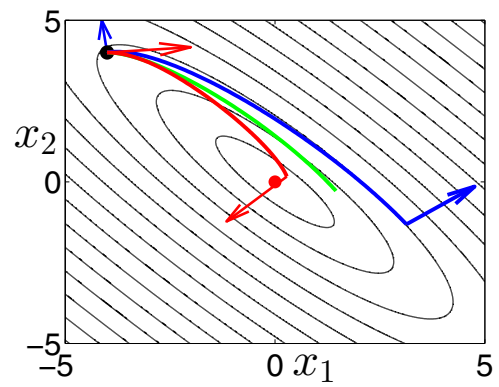

Fig. 1. Optimal trajectories for a linear system with stable focus $\left(\dot{x}_{1}=\right.$ $3 x_{1}+4 x_{2}, \dot{x}_{2}=-4 x_{1}-4 x_{2}$ ). For the optimal escape problem (blue curve), the optimal time delay with respect to the unforced trajectory (green curve) is $\Delta t^{*}=1.080$. For the optimal convergence problem (red curve), the optimal time delay is $\Delta t^{*}=2.068$. The arrows are the controls $e^{*}(0)$ and $e^{*}(T)$. The black curves are the isostables. The red and black dots are the stable equilibrium and the initial condition, respectively. (Parameters: $x_{0}=[4,4], \bar{u}=1, T=1$.)

of high concentration. In this context, the optimal control results from the trade-off between (i) a high instantaneous variation of $\phi$ (control perpendicular to the isostables) and (ii) the expectation of a higher average variation of $\phi$ (control toward regions of higher concentration of isostables). This trade-off depends on the magnitude $\bar{u}$. If $\bar{u} \ll|F|$, the control cannot drive the trajectory toward regions of high gradient $\nabla_{x}|\phi|$ and the option (i) is preferred so that we recover the result of Section III-C.1. This trade-off phenomenon, also observed with nonlinear systems, is clearly shown in Fig. 4(b) in Section IV.

3) Nonlinear systems: For nonlinear systems, the isostables are computed through (7) and the optimal control problem is solved numerically. Optimal trajectories are illustrated in Fig. 2 (convergence to a sink) and in Fig. 3 (escape from a stable focus). In Fig. 2, we note that the isostable $\phi=0$ is a curve: it does not only contain the equilibrium, but also corresponds to trajectories characterized by a rate of convergence $\lambda_{2}<\lambda_{1}$, which are preferable over any other free motion trajectories.

Performances of the optimal control (12) and the local control (10) perpendicular to the isostables (see Section IIIA) are compared. In Fig. 2, the two methods yield similar results, an observation which is explained by the fact that $\bar{u}$ is small with respect to the vector field (so that the local control is almost optimal). In contrast, the optimal control in Fig. 3 yields much better results than the local control. In this case, the two controls are completely different (see e.g. the initial directions $e^{*}(0)$ and $e^{(*)}(0)$ ). Taking advantage of the global knowledge of the geometry of the isostables, the optimal control steers the trajectory toward the equilibrium to reach a region (on the other side of the equilibrium) where the isostables are concentrated and where the escape is more efficient. The optimal (but counter-intuitive) control contrasts with the local control which tends to maximize the instantaneous increase of $|\phi|$ and never points toward the equilibrium. Note that this phenomenon is not observed in the case of optimal convergence.

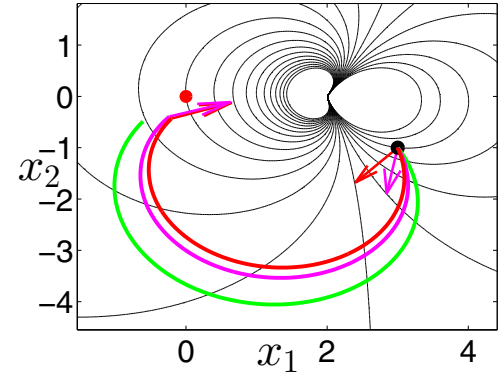

Fig. 2. Optimal convergence problem for the system $\dot{x}_{1}=-2 x_{1}+x_{1}^{2}-x_{2}^{2}$, $\dot{x}_{2}=-2.5 x_{2}+2 x_{1} x_{2}$ (optimal trajectory in red, local control in magenta, free motion in green). The time delay obtained with the optimal control $\left(\Delta t^{*}=0.362\right)$ is similar to the time of delay obtained with the local control $\left(\Delta t^{(*)}=0.348\right)$. The arrows are the initial and final controls. The black curves are the isostables. The red and black dots are the stable equilibrium and the initial condition, respectively. (Parameters: $x_{0}=[-3,1], \bar{u}=1$, $T=1$.)

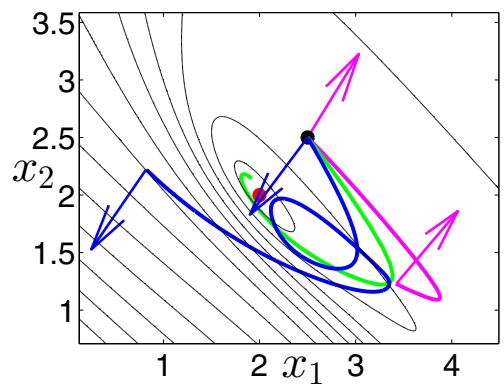

Fig. 3. Optimal escape problem for the system $\dot{x}_{1}=2-4 x_{1}+x_{1}^{2} x_{2}-x_{1}$, $\dot{x}_{2}=4 x_{1}-x_{1}^{2} x_{2}$ (optimal trajectory in blue, local control in magenta, free motion in green). The time delay obtained with the optimal control $\left(\Delta t^{*}=\right.$ $5.120)$ is much larger than the time of delay obtained with the local control $\left(\Delta t^{(*)}=2.685\right)$. The arrows are the initial and final controls. The black curves are the isostables. The red and black dots are the stable equilibrium and the initial condition, respectively. (Parameters: $x_{0}=[2.5,2.5], \bar{u}=1$, $T=3$.)

\section{MAGNitude AND DURATION OF THE CONTROL: OPTIMAL STRATEGIES}

In this section, we consider that the magnitude and duration of the control can be chosen and we discuss the strategy which maximizes the time delay (8). Assuming that the net effect of the control after time $T$ is fixed, we impose the constraint $\bar{u} T=\alpha$. (The constant $\alpha>0$ corresponds to the maximum deviation of the trajectory of the controlled system with respect to the trajectory of the unforced system.) If there is no (lower) bound on $\bar{u}$, we show that the optimal strategy is to prefer a long control with small magnitude rather than a short impulsive control with high magnitude.

The case of a linear system with $\lambda_{1}$ real can be studied analytically. In the case of optimal convergence, considering (20) with $\bar{u}=\alpha / T$, we can easily see that there exists a value $T_{c r}>0$ such that $\phi\left(x^{*}\left(T_{c r}\right)\right)=0$. It follows that, for values $T>T_{c r}\left(\bar{u}<\alpha / T_{c r}\right)$, the control pushes the trajectory onto the isostable $|\phi|=0$, thereby yielding an infinite time delay (8). Choosing $\bar{u}$ small and $T$ large is therefore the optimal strategy. In the case of optimal escape, there is no value 
$T_{c r}$. In addition, the derivation of (8) with (20) with respect to $T$ shows that the optimal delay increases as the time $T$ increases, for $T \gg 1$. In this case, the optimal strategy is also achieved with a large value $T$ and a small magnitude $\bar{u}$.

For a nonlinear system with $\lambda_{1}$ real, the above results still hold since the trajectory driven by a weak control converges to the neighborhood of the equilibrium, where the dynamics is well approximated by a linear dynamics and where the weak control is the optimal strategy (see Fig. 4). In the case of systems with $\lambda_{1}$ complex, numerical simulations show that large optimal time delays are also obtained with a weak control (see Fig. 5). Hence, choosing $\bar{u}$ small and $T$ large is always the optimal strategy. This corresponds to the local control (10).

If a weak control is not feasible (e.g. if we have the additional constraint $\bar{u} \in\left[\bar{u}_{\min }, \bar{u}_{\max }\right]$ ), the presence of (local) minima and maxima in Fig. 4(a) and 5(a) indicates that other combinations $(T, \bar{u})$ might be optimal (and some combinations should be avoided). For instance, if the control duration must be very short, an impulsive control $(T \rightarrow 0$, $\bar{u} \rightarrow \infty$ ) should be the optimal strategy.

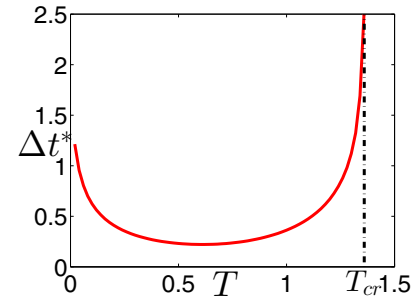

(a)

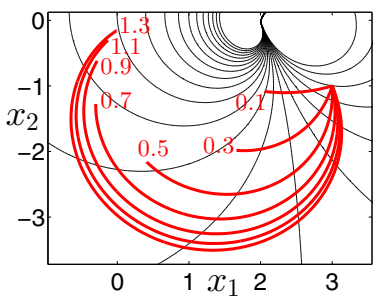

(b)
Fig. 4. Optimal strategy for the convergence problem with the system considered in Fig. 2. (a) The maximum optimal time delay is obtained at $T_{c r} \approx 1.36$ (weak control). Note that the impulsive control $(T \rightarrow 0)$ also yields a large time delay. Intermediate values give the worst results. (b) Optimal trajectories obtained for different values $T$ and $\bar{u}=1 / T$. The number at the end of each trajectory corresponds to $T$. (Parameters: $x_{0}=[-1,3], \alpha=1$.)

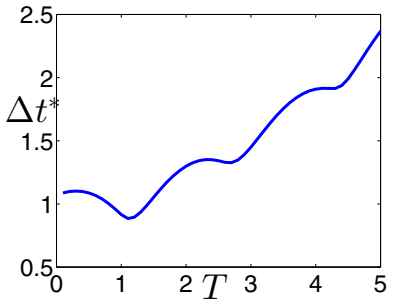

(a)

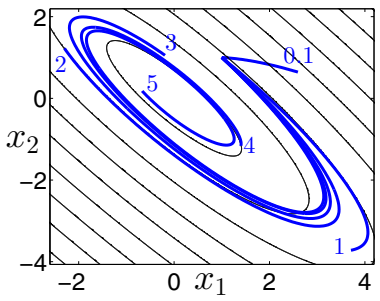

(b)
Fig. 5. Optimal strategy for the escape problem with the system considered in Fig. 1. (a) Large optimal time delays are obtained with large values $T$. We also remark the presence of local minima and maxima. (b) Optimal trajectories obtained for different values $T$ and $\bar{u}=1 / T$. The number at the end of each trajectory corresponds to $T$. (Parameters: $x_{0}=[2,2]$, $\alpha=1$.)

\section{CONClusion}

We have proposed and studied a finite-horizon optimal control problem which corresponds to the optimal convergence to (or escape from) an equilibrium and which is also adapted to a control of short duration. In this context, we have shown that the concept of isostables is of paramount importance, since it is necessary to define an appropriate end cost function for the problem.

In the case of weak and strong control inputs, exact optimal solutions are directly related to the geometry of the isostables. In addition, a so-called "local" control perpendicular to the isostables can be considered when the global isostables or the vector field are unknown. In the other cases, the optimal control problem is solved numerically through Pontryagin's maximum and the solution is not directly related to the isostables. Finally, we have shown that an optimal strategy consists in choosing a small control magnitude in favor of a long control duration, a situation which corresponds to the local control.

The results presented in the paper are limited to systems with a stable equilibrium. The optimal control problem could be extended to other types of attractors, in which case the notion of isostables might need to be generalized. In the case of limit cycles, a similar problem using the so-called isochrons (instead of the isostables) could be investigated and related to existing results of time-optimal control [8].

\section{ACKNOWLEDGMENTS}

This work was performed while A. Mauroy held a Back to Belgium Grant of the Belgian Federal Science Policy (BELSPO).

\section{REFERENCES}

[1] Y. F. Golubev. A time-optimal control of steering an unstable rod. Journal of Computer and Systems Sciences International, 47(5):709717, 2008.

[2] D. E. Kirk. Optimal control theory: an introduction. Courier Dover Publications, 2012.

[3] A. Lasota and M. C. Mackey. Chaos, Fractals, and Noise: stochastic aspects of dynamics. Springer-Verlag, 1994.

[4] A. Mauroy and I. Mezić. A spectral operator-theoretic framework for global stability. In Proceedings of the 52th IEEE Conference on Decision and Control, pages 5234-5239, December 2013.

[5] A. Mauroy, I. Mezić, and J. Moehlis. Isostables, isochrons, and Koopman spectrum for the action-angle representation of stable fixed point dynamics. Physica D: Nonlinear Phenomena, 261:19-30, October 2013.

[6] D. Q. Mayne and W. R. Schroeder. Robust time-optimal control of constrained linear systems. Automatica, 33(12):2103-2118, 1997.

[7] I. Mezić. Analysis of fluid flows via spectral properties of Koopman operator. Annual Review of Fluid Mechanics, 45, January 2013.

[8] A. Nabi and J. Moehlis. Time optimal control of spiking neurons. Journal of mathematical biology, 64(6):981-1004, 2012.

[9] L. S. Pontryagin. Mathematical theory of optimal processes. CRC Press, 1987.

[10] A. Rabinovitch and I. Rogachevskii. Threshold, excitability and isochrones in the Bonhoeffer-van der Pol system. Chaos, 9(4):880886, 1999.

[11] S. A. Reshmin and F. L. Chernous'ko. A time-optimal control synthesis for a nonlinear pendulum. Journal of Computer and Systems Sciences International, 46(1):9-18, 2007.

[12] B. Rhoads, I. Mezić, and A. Poje. Minimum time feedback control of autonomous underwater vehicles. In Proceedings of the 49th IEEE Conference on Decision and Control, pages 5828-5834. IEEE, 2010. 